\title{
Análise da adiposidade subcutânea durante o ciclo menstrual
}

\author{
Analysis of subcutaneous adiposity during the menstrual cicle
}

Joaquim Huaina Cintra Andrade, ${ }^{1}$ Arthur Monteiro da Silva, ${ }^{1}$ Francisca Thalia Rodrigues Amorim, ${ }^{2}$

Romário Pinheiro Lustosa, Natália Macedo Uchoa, ${ }^{3}$ Francisco Nataniel Macedo Uchoa ${ }^{4}$

'Universidade Estadual do Ceará, Fortaleza, CE, Brasil.

2Centro Universitário Estácio do Ceará, Fortaleza, CE, Brasil.

3Universidade de Fortaleza (UNIFOR), Fortaleza, CE, Brasil.

¿Universidade de Trás-os-Montes e Alto Douro - UTAD, Portugal.

Recebido em: 10/09/2016 / Aceito em: 17/02/2017 / Publicado em: 31/03/2017

joaquimcintra@hotmail.com

\section{RESUMO}

Objetivo: analisar isoladamente o componente adiposo subcutâneo durante o ciclo menstrual. Método: a amostra foi composta por 30 mulheres saudáveis com idade entre 18 e 25 anos, com ciclo menstrual regular e que não utilizavam anticoncepcional oral ou injetável no período do estudo, na cidade de Fortaleza-CE, Brasil. A adiposidade corporal foi predita a partir da quantificação do somatório de seis dobras cutâneas (S ADIP). Essa variável foi utilizada no cálculo do escore de proporcionalidade ( $Z$ ADIP), subsidiando a realização do cálculo da massa adiposa absoluta (M ADIP). Resultados: a média de idade foi de 21,8 anos e de estatura 162,2 $\mathrm{cm}$. Considerando a disposição de adiposidade por dobra cutânea, durante as três fases do ciclo menstrual, as dobras tricipital, subescapular, abdominal e espinal apresentaram maior média na fase lútea $(14,8 ; 11,9$; 19,$5 ; 10,6$ respectivamente); a dobra coxa apresentou valores de médias estatisticamente semelhantes nas fases folicular e ovulatória $(20,1)$ e a dobra perna apresentou maior média na fase folicular $(13,4)$. Analisando a prevalência do maior somatório de dobras cutâneas e a relação com a maior quantificação de massa adiposa absoluta, constatou-se que a fase folicular apresentou a maior média de somatório de dobras $(89,6)$ e de massa adiposa $(19,3)$. Considerações finais: os dados encontrados revelam que, durante as fases do ciclo menstrual, não há diferença significativa no somatório das dobras cutâneas e de massa adiposa absoluta. Este fato sugere que as alterações hormonais ocorridas durante o ciclo não estão diretamente associadas com o fator de variabilidade da adiposidade corporal.

Palavras-chave: Ciclo Menstrual; Composição Corporal; Antropometria; Tecido Adiposo.

\section{ABSTRACT}

Objective: to analyze separately the subcutaneous adipose component during the menstrual cycle. Method: sample consisted of 30 healthy women aged between 18 and 25 years with regular menstrual cycle and who did not use oral or injectable contraceptive during the study period in the city of Fortaleza, Brazil. The body adiposity was predicted from the quantification of the sum of six skinfold (S ADIP). This variable was used to calculate the proportionality score (Z ADIP) subsidizing the completion of the calculation of absolute adipose mass (M ADIP). Results: the average age was 21.8 years and height $162.2 \mathrm{~cm}$. Considering the array of adiposity by skinfold during the three phases of the menstrual cycle, the folds triceps, subscapularis, abdominal and spinal had a higher average in the luteal phase 114.8; $11.9 ; 19.5 ; 10.6$ respectively); skinfold thigh presented mean values statistically similar in the stage follicular and ovulatory phases (20.1) and the leg folds had a higher average in the follicular phase (13.4). Analyzing the prevalence of greater sum of skinfolds and the relationship with the largest absolute quantification of adipose mass, it was found that the follicular phase had the highest average skinfold sum (89.6) and adipose mass (19.3). Closing remarks: the data found reveal that during of the menstrual cycle there is no significant difference in the sum of skinfolds and absolute adipose mass. This fact suggests that hormonal changes during the cycle are not directly associated as a factor in the variability of body adiposity.

Keywords: Menstrual Cycle; Body Composition; Anthropometry; Adipose Tissue. 


\section{INTRODUÇÃO}

A menstruação é um sangramento vaginal periódico que começa na menarca e termina com a menopausa. Trata-se do final dos eventos causados pela ação integrada do eixo hipotálamo-hipófise-ovário (HHO), que determinam as modificações fisiológicas do organismo feminino, visando prepará-lo para reprodução. O ciclo menstrual é o conjunto destas modificações, iniciando-se no primeiro dia da menstruação. ${ }^{1}$

O ciclo menstrual divide-se em três fases: folicular, lútea e ovulatória. ${ }^{2}$ No início da fase folicular, os níveis plasmáticos de estrógenos e de progesterona estão baixos, pelo mecanismo de feedback negativo, o FSH (hormônio folículo estimulante) plasmático começa a elevar-se já durante a fase de menstruação, tendo seu pico perto da metade da fase proliferativa. Nessa fase, o organismo feminino está sob a influência de grandes concentrações de estrógenos, que facilitam mais o desenvolvimento do folículo, aumentando também a camada granulosa e a ligação de $\mathrm{FSH}$, a receptores foliculares. Durante essa ação estrogênica, o endométrio uterino apresenta alta atividade mitótica do epitélio e do estroma. ${ }^{2,3}$

A ovulação na mulher com ciclo menstrual normal de 28 dias, ocorre 14 dias após o início da menstruação, dividindo as fases proliferativa e secretória; ela não ocorre por um aumento da pressão intrafolicular, mas sim parece ocorrer uma digestão enzimática, com dissociação da camada celular granulosa, mudanças degenerativas no epitélio germinativo e enfraquecimento das camadas tecais, fazendo com que a pressão normal seja suficiente para causar a expulsão do óvulo. ${ }^{3}$

Após a ovulação, o folículo que liberou o óvulo começa a luteinizar-se sob a ação do LH (Hormônio Luteinizante), os níveis de progesterona aumentam drasticamente, tendo o nível máximo atingido 5 a 6 dias após a ruptura do folículo. Aumenta o conteúdo epitelial de glicogênio, há uma invasão de linfócitos no endométrio e aumenta a vascularização do endométrio para a implantação do óvulo fertilizado. Ocorre, em especial, uma inibição da contratilidade uterina. Assim, se não houver a fecundação, o corpo lúteo começa a degenerar-se, sendo substituído por um tecido conjuntivo acelular e avascular denominado corpo albicans. ${ }^{2,3}$

Com a regressão do corpo lúteo, os níveis de estrogênios e progesterona, particularmente de progesterona, diminuem, causando assim a menstruação. A menstruação tem uma duração variável entre 3 a 7 dias, e durante esse período é eliminado um volume de 70 a $150 \mathrm{ml}$ de liquido incoagulável, pela presença de fibrinolisina. Ainda, durante a menstruação, os níveis baixos de hormônios sexuais fazem com que a hipófise comece a liberar $\mathrm{FSH}$, por mecanismo retroalimentação negativa, que atuando sobre um novo folículo, dá início a um novo ciclo. ${ }^{3}$

O estrogênio é secretado pelos ovários durante o ciclo reprodutivo sendo responsável pela estimulação do estrato basal do endométrio, propiciando o desenvolvimento funcional dessa estrutura; pelas diferenças físicas da mulher e do homem (desenvolvimento das mamas, alargamento dos quadris e o estreitamento pélvico) e ainda induz o tecido adiposo a concentrar-se na mulher em áreas como os quadris e coxas, dando-Ihes o arredondamento típico do sexo feminino. ${ }^{2,3}$ Entretanto, devido à distribuição e disposição de tecido adiposo subcutâneo ser caracterizada pela especificidade do sexo e individualidade biológica, respectivamente, viabiliza importância da avaliação da composição corporal devido ao fato de que a massa corporal isoladamente não é considerada um bom parâmetro para a identificação do excesso ou déficit dos componentes corporais. ${ }^{4}$

As informações apresentadas na literatura evidenciam que as espessuras de dobras cutâneas são as medidas antropométricas mais comumente utilizadas na análise dos parâmetros da composição corporal alicerçadas na observação de que indivíduos adultos na faixa etária entre 20 e 50 anos de idade, apresentam uma proporção de $50 \%$ a $70 \%$ da gordura corporal que está localizada subcutaneamente e algumas dobras cutâneas têm mostrado relação com a adiposidade corporal total. ${ }^{5,6}$ Entretanto, há uma errônea utilização terminológica em associar os conceitos de gordura e tecido adiposo como sinônimos, sendo que estes se constituem como duas estruturas biológicas divergentes. ${ }^{7}$

A gordura é definida como a totalização de lipídeos existentes no organismo, sendo estes, substâncias químicas, como por exemplo; triglicerídeos, fosfolipídios e esteroides, os quais são indissolúveis na água e dissolúveis em soluções orgânicas como o éter. Por consequente, o tecido adiposo é constituído por células adiposas (adipócitos), fluidos extracelulares, endotélio vascular, colágeno e fibras elastinas. ${ }^{7}$

Algumas mulheres, especialmente aquelas cujo peso corporal oscila nos ciclos menstruais, podem ter estimativas significativamente diferentes de densidade corporal e percentual de gordura quando pesadas hidrostaticamente em momentos diferentes de seus ciclos. ${ }^{8}$

Contudo, acredita-se na possibilidade das mudanças fisiológicas decorrentes do ciclo menstrual alterar as medidas antropométricas e de composição corporal. ${ }^{9}$ Deste modo, o presente estudo tem como objetivo analisar isoladamente o componente adiposo subcutâneo durante o ciclo menstrual.

\section{MÉTODO}

O presente estudo é caracterizado como descritivo, transversal e com abordagem quantitativa. O referido estudo foi desenvolvido no Núcleo de Cineantropometria e Performance Esportiva ${ }^{\circledR}$ consultório cineantropométrico da Academia Boa Forma ${ }^{\circledR}$ localizada na Rua José Mendonça, 1047, Bairro Genibaú na cidade de Fortaleza (CE).

A amostra foi composta por 30 mulheres saudáveis, com idades entre 18 e 25 anos e média 21,8 \pm 2,2 anos. Como critério de inclusão, as voluntárias não deveriam fazer o uso de anticoncepcionais no período do estudo e possuírem o ciclo menstrual com regularidade periódica de aproximadamente 28 dias, sendo, portanto, excluídas da amostra aquelas que reportaram histórico de qualquer distúrbio relacionado ao sistema endócrino e que engravidarem no período do estudo.

Todas as voluntárias assinaram um Termo de Consentimento Livre e Esclarecido que atende à Resolução de $n^{\circ} 466 / 2012$ do Conselho Nacional de Saúde (CNS) do Ministério da Saúde (MS), relacionado à pesquisa envolvendo seres humanos.

Os dados coletados foram organizados em uma planilha eletrônica elaborada através de um aplicativo do software Microsoft Excel ${ }^{\circledR} 2016$, versão 1.19 (Micro- 
soft Corporation ${ }^{\odot}$, EUA), instalado em um iPad ${ }^{\circledR}$ Mini (Apple ${ }^{\odot}$ Inc., EUA), estruturada com informações referentes à identificação das voluntárias e registros das variáveis antropométricas.

O horário das avaliações foi padronizado para cada voluntária de acordo com a primeira visita. Todas as integrantes realizaram três visitas ao consultório de forma aleatória (indivíduo $x$ fase do ciclo), sendo submetidas aos procedimentos de coleta das medidas antropométricas. Com a objetividade de minimizar erros sistemáticos foi solicitado o esvaziamento da bexiga urinária previamente à realização dos procedimentos, abstenção da prática de exercícios físicos e de ingestão de bebidas alcoólicas, nas 24 horas precedentes.

Para determinação das fases em relação ao dia do ciclo menstrual foi utilizado o critério proposto por Wojtys et al. ${ }^{10} \mathrm{em}$ que a fase folicular inicia no primeiro dia $\left(1^{\circ}\right)$ do ciclo (início da menstruação) e perdura até o nono dia $\left(9^{\circ}\right)$, a fase ovulatória ocorre entre o décimo $\left(10^{\circ}\right)$ e décimo quarto $\left(14^{\circ}\right)$ dia e a fase lútea inicia a partir do décimo quinto $\left(15^{\circ}\right)$ dia e dura até o final do ciclo menstrual.

Foram selecionadas e mensuradas as medidas antropométricas da massa corporal, utilizando uma balança eletrônica (Toledo ${ }^{\circledR}$, São Paulo, Brasil), com precisão de $50 \mathrm{~g}$, estatura utilizando um estadiômetro (Sanny ${ }^{\circledR}$, São Paulo, Brasil) com resolução em milímetros e seis dobras cutâneas (tricipital, subescapular, supraespinal, abdominal, coxa e panturrilha), utilizando um plicômetro científico Cescorf Premier (Cescorf ${ }^{\circledR}$, Porto Alegre, Brasil) com resolução em décimos de milímetros e compressibilidade de $10 \mathrm{~g} / \mathrm{mm}^{2}$. Destaca-se que as variáveis antropométricas foram mensuradas no hemicorpo direito, utilizando o critério de triplicata para obter o valor de medida mediana em concordância com as diretrizes e padronizações técnicas do International Standards For Anthropometric Assessment, ${ }^{11}$ sendo, portanto, executadas por um antropometrista com experiência prévia e acreditação (nível dois) concedida pela International Society For The Advancement Of Kinanthropometry (ISAK).

A adiposidade subcutânea foi predita a partir da quantificação do somatório de seis dobras cutâneas (S ADIP) de regiões anatômicas periféricas e centrais, portanto, analisando de forma fidedigna a topografia adiposa. Essa variável foi utilizada no cálculo do escore de proporcionalidade (Z ADIP), subsidiando a realização do cálculo da massa adiposa absoluta (M ADIP). ${ }^{12}$ As equações para determinação do escore de proporcionalidade e predição da massa adiposa absoluta em análise no presente estudo foram apresentadas no quadro 1.

Para a análise dos cálculos e interpretação dos resultados foi elaborado um banco de dados por meio de uma planilha eletrônica e realizados procedimentos de análises estatísticas descritivas e inferenciais utilizando o software SPSS $^{\circledR}$, versão 17.0 (SPSS Inc., Chicago, IL, EUA).

\section{RESULTADOS}

A disposição de adiposidade por dobra cutânea, considerando as três fases do ciclo menstrual (Tabela 1), caracterizou-se na relevância de que as dobras tricipital, subescapular, abdominal e espinal apresentaram maior média na fase lútea $(14,8 ; 11,9 ; 19,5 ; 10,6$

Quadro 1 - Equações para determinação do somatório de dobras cutâneas, escore de proporcionalidade e predição da massa adiposa absoluta. ${ }^{12}$

\begin{tabular}{|l|l|}
\hline Variáveis & Equação \\
\hline Somatório de Dobras Cutâneas & S ADIP $=$ TR + SB + SE + AB + CX + PN \\
\hline Escore de Proporcionalidade & Z ADIP $=[S$ ADIP $\bullet(170,18 / H)-116,41] / 34,79$ \\
\hline Massa Adiposa Absoluta & M ADIP $(\mathrm{kg})=[($ Z ADIP $\bullet 5,85)+25,6] /(170,18 / H)^{3}$ \\
\hline
\end{tabular}

Legenda: $S A D I P=$ Somatório de 6 dobras cutâneas; $T R=$ Tricipital; $S B=$ Subescapular; $S E=$ Supraespinal; $A B=A b d o m i n a l ; C X=C o x a ; P N=P e r n a ; Z A D I P=E s c o r e$ de proporcionalidade $H$ = Estatura em centímetros; $M A D I P=$ Massa adiposa absoluta.

Tabela 1 - Disposição adiposa por dobra cutânea nas fases do ciclo menstrual.

\begin{tabular}{|c|c|c|c|c|c|}
\hline \multirow[t]{2}{*}{ Dobras } & \multirow[t]{2}{*}{ Fase do Ciclo } & \multirow[t]{2}{*}{ Média } & \multirow[t]{2}{*}{ DP } & \multicolumn{2}{|c|}{ Disposição adiposa } \\
\hline & & & & Diferença maior-menor & Diferença L-F \\
\hline \multirow[t]{3}{*}{ Triciptal } & $\mathrm{F}$ & 14,517 & 2,1509 & 0,443 & 0,313 \\
\hline & $\mathrm{O}$ & 14,387 & 2,2695 & & \\
\hline & $\mathrm{L}$ & 14,830 & 2,0996 & & \\
\hline \multirow[t]{3}{*}{ Subescapular } & $\mathrm{F}$ & 11,737 & 2,8524 & 0,17 & 0,17 \\
\hline & $\mathrm{O}$ & 11,857 & 2,8883 & & \\
\hline & $\mathrm{L}$ & 11,907 & 2,9804 & & \\
\hline \multirow[t]{3}{*}{ Espinal } & $\mathrm{F}$ & 10,497 & 4,8890 & 0,33 & 0,106 \\
\hline & $\mathrm{O}$ & 10,273 & 4,2080 & & \\
\hline & $\mathrm{L}$ & 10,603 & 4,9288 & & \\
\hline \multirow[t]{3}{*}{ Abdominal } & $\mathrm{F}$ & 19,473 & 4,8731 & 0,547 & 0,067 \\
\hline & $\mathrm{O}$ & 18,993 & 5,3669 & & \\
\hline & $\mathrm{L}$ & 19,540 & 5,2880 & & \\
\hline \multirow[t]{3}{*}{ Coxa } & $\mathrm{F}$ & 20,107 & 2,9909 & 0,538 & $-0,532$ \\
\hline & $\mathrm{O}$ & 20,113 & 3,9752 & & \\
\hline & $\mathrm{L}$ & 19,575 & 3,1106 & & \\
\hline \multirow[t]{3}{*}{ Perna } & $\mathrm{F}$ & 13,477 & 2,8364 & 0,564 & $-0,24$ \\
\hline & $\mathrm{O}$ & 12,913 & 2,7308 & & \\
\hline & $\mathrm{L}$ & 13,237 & 2,5594 & & \\
\hline
\end{tabular}

Legenda: DP: Desvio-Padrão; F: Fase Folicular; O: Fase Ovulatória; L: Fase Lútea 
respectivamente); a dobra coxa apresentou valores de médias estatisticamente semelhantes nas fases folicular e ovulatória $(20,1)$ e a dobra perna apresentou maior média na fase folicular $(13,4)$.

Analisando a prevalência do maior somatório de seis dobras cutâneas (Tabela 2) e a relação com a maior quantificação de massa adiposa absoluta (Tabela 3), nas três fases do ciclo menstrual, constatou-se que a fase folicular apresentou a maior média de somatório de dobras $(89,6)$ e de massa adiposa $(19,3)$. No entanto, destaque similaridades para as médias de S ADIP e $M$ ADIP, nas fases folicular e lútea (Tabela 2 e 3 ).

Tabela 2 - Média do somatório de seis dobras (S ADIP) em cada fase do ciclo menstrual.

\begin{tabular}{|c|c|c|c|c|}
\hline \multirow[t]{2}{*}{ Fase do Ciclo } & \multicolumn{2}{|c|}{ S ADIP } & \multirow{2}{*}{$\begin{array}{c}\text { Valor } \\
\text { Mínimo }\end{array}$} & \multirow{2}{*}{$\begin{array}{c}\text { Valor } \\
\text { Máximo }\end{array}$} \\
\hline & Média & DP & & \\
\hline Folicular & 89,647 & 14,1038 & 72,8 & 120,0 \\
\hline Ovulatória & 87,88 & 15,1354 & 70,2 & 122,3 \\
\hline Lútea & 89,454 & 15,1745 & 70,3 & 125,3 \\
\hline
\end{tabular}

Tabela 3 - Média da massa adiposa absoluta (M ADIP) em cada fase do ciclo menstrual.

\begin{tabular}{|c|c|c|c|c|}
\hline \multirow[t]{2}{*}{ Fase do Ciclo } & \multicolumn{2}{|c|}{ M ADIP } & \multirow{2}{*}{$\begin{array}{c}\text { Valor } \\
\text { Mínimo }\end{array}$} & \multirow{2}{*}{$\begin{array}{c}\text { Valor } \\
\text { Máximo }\end{array}$} \\
\hline & Média & DP & & \\
\hline Folicular & 19,336 & 3,4265 & 13,7 & 27,0 \\
\hline Ovulatória & 19,082 & 3,6167 & 12,9 & 26,9 \\
\hline Lútea & 19,238 & 3,6412 & 13,4 & 27,9 \\
\hline
\end{tabular}

\section{DISCUSSÃO}

Quanto à distribuição do tecido adiposo subcutâneo, nas regiões anatômicas periféricas e centrais, houve preeminência na fase lútea do ciclo menstrual. Durante essa fase, quatro dobras cutâneas demonstraram predominância, sendo uma dobra periférica (tricipital) e três dobras centrais (subescapular, abdominal e espina/). Na fase folicular, duas dobras cutâneas periféricas (coxa e perna) demonstraram predominância, contudo, na fase ovulatória, apenas a dobra periférica coxa obteve relevância. Portanto, os dados encontrados corroboram com Guedes e Guedes ${ }^{7}$ que afirmam sobre o tecido adiposo subcutâneo não se distribuir uniformemente por todo o corpo humano, sendo, deste modo, necessária a coleta das medidas de espessura das dobras cutâneas em uma variabilidade de regiões anatômicas para obter uma análise mais fidedigna, quanto à sua disposição.

Desta forma, os dados encontrados revelam que, durante as fases do ciclo menstrual, não há diferença significativa no somatório das dobras cutâneas e de massa adiposa absoluta, apresentando semelhança com os achados de Gleichauf e Roe, ${ }^{13}$ que avaliaram 26 mulheres que não utilizavam contraceptivos orais ou injetáveis e encontrou diferenças significativas na massa corporal ( $p>0,05)$ e massa livre de gordura ( $p>0,05$ ), nas diferentes fases do ciclo, contudo, o percentual de adiposidade $(p=0,05)$ não apresentou divergências.

Em conformidade com os achados encontrados, no estudo de Teixeira et al. ${ }^{14}$ que avaliaram a flexibilidade e a composição corporal de 82 mulheres, aparentemente saudáveis, com idades entre 18 e 40 anos. Avaliando-as com uso de contraceptivos hormonais e sem. Os autores também não encontraram diferenças significativas na composição corporal (percentual de gordura e massa magra) entre as fases do ciclo menstrual ( $p>0,05)$.

Frisch e Mcarthur ${ }^{15}$ demonstraram que, para haver um funcionamento adequado do ciclo menstrual, é necessário um mínimo de $22 \%$ de adiposidade corporal. No presente estudo, foi encontrado um valor médio de $(19,336),(19,082),(19,238)$ nas fases folicular, ovulatória e lútea, respectivamente. Estes autores associaram essa quantidade de tecido adiposo com a produção de estrógeno ovariano. Portanto, em mulheres com percentual de adiposidade abaixo dos níveis estabelecidos, a produção de estrógeno ovariano e a regularidade do ciclo menstrual seriam comprometidas.

\section{CONSIDERACִÕES FINAIS}

No presente estudo, não foram observadas no grupo analisado diferenças significativas na adiposidade corporal durante o ciclo menstrual. Este fato pode sugerir que as alterações hormonais ocorridas, durante o ciclo, não estão diretamente associadas como fator de variação do tecido adiposo subcutâneo.

A ausência de resultados consistentes deve-se ao fato de que as participantes desse estudo estiveram adequadas aos critérios de inclusão exigidos, portanto, divergindo, de fatores comumente existentes na fisiologia feminina, como por exemplo, a presença de ciclos menstruais irregulares e prolongados (oligomenorreia); predisposição genética; desordens alimentares; distúrbios dos hormônios tireoidianos e a supressão dos hormônios reprodutivos, através de utilização de contraceptivo oral ou injetável que podem estar relacionados com a presença de variabilidade na disposição adiposa subcutânea, sugerindo a possibilidade de ocasionar desequilíbrio na adiposidade corporal feminina.

Contudo, destaca-se a utilização do somatório de dobras cutâneas (S ADIP) como um procedimento viável e não invasivo para a predição da adiposidade corporal, sugerindo, por consequente, que a variabilidade da quantificação do somatório está diretamente relacionada ao excesso ou déficit do componente tecidual adiposo, todavia, para que haja confiabilidade nessa análise preditiva é necessário que a seleção das dobras cutâneas se estabeleça proporcionalmente entre as regiões anatômicas centrais e periféricas.

$\mathrm{Na}$ literatura específica, são evidenciados estudos sobre as alterações do componente residual, tendo como variabilidade o aporte hídrico durante o ciclo menstrual, entretanto, devido à baixa produção científica relacionada ao componente adiposo, torna-se necessário à condução de estudos na área de Cineantropometria Morfológica, utilizando as variáveis de predição dos tecidos corporais propostos pelo o método de cinco componentes como um parâmetro de análise fidedigna da composição corporal, durante o ciclo menstrual.

\section{REFERÊNCIAS}

1. Deligeoroglou E, Tsimaris P. Menstrual disturbances in 
puberty. Best Pract Res Clin Obstet Gynaecol 2010; 24(2):157-71. doi: 10.1016/j.bpobgyn.2009.11.001

2. Guyton AC, Hall JE. Tratado de fisiologia médica. 2011, 12. ed. Rio de Janeiro: Guanabara Koogan.

3. Douglas CR. Tratado de fisiologia aplicado às ciências médicas. 2006, 6. Ed. Rio de Janeiro: Guanabara Koogan.

4. Costa RF. Avaliação Física. São Paulo. Fitness Brasil Collection, 1996.

5. Lohman TG. Advances in body composition assessment. Champaign, Illinois, Human Kinetics, 1992.

6. Lohman TG. Skinfolds and body density and their relation to body fatness: a review. Human Biology 1981;53(2):181-225.

7. Guedes DPG, Guedes JERP. Controle do peso corporal: Composição corporal, atividade física e nutrição. 2003, 2. ed. Rio de Janeiro: Shape.

8. Heyward HV. Avaliação física e prescrição de exercício: técnicas avançadas. Porto Alegre: Editora Artmed, 2010, 6. Ed.

9. Teixeira ALS, Júnior FW, Moraes EM, Alves BA, Dias MR. Efeito das diferentes fases do ciclo menstrual na composição corporal de universitárias. RBPFEX 2012;6(35):428-32.

10. Wojtys EM, Huston LJ, Lindenfeld TN, Hewett TE, Greenfield
MLVH. Association between the menstrual cycle and anterior cruciate ligament injuries in female athletes. The American Journal of Sports Medicine 1998;26(5):614-19. doi: $10.1177 / 03635465980260050301$.

11. Stewart A, Marfell-Jones M, Olds T, Ridder H. International Standards For Anthropometric Assessment. ISAK, 2011.

12. Ross W, Kerr D. Fraccionamiento de laMasa Corporal: UnNuevo Método para Utilizar en Nutrición, Clínica y Medicina Deportiva. Apunts 1991;28(109):175-87.

13. Gleichauf CN, Roe DA. The menstrual cycle's effect on the reliability of bioimpedance measurements for assessing body composition. Am J Clin Nutr 1989;50(5):903-7.

14. Teixeira ALS, Fernandes Júnior W, Marques FAD, Lacio ML, Dias MRC. Influência das diferentes fases do ciclo menstrual na flexibilidade de mulheres jovens. Rev Bras Med Esporte 2012;18(6):361-4. doi: 10.1590/S151786922012000600002.

15. Frisch, R. E; MCarthur, J. W. Menstrual cycles: fatness as a determinant of minimum weight for height necessary for their maintenance or onset. Science 1974;185:949-51. doi: 10.1126/science.185.4155.949.

Como citar: ANDRADE, Joaquim Huaina Cintra et al. Análise da adiposidade subcutânea durante o ciclo menstrual. Cinergis, Santa Cruz do Sul, v. 18, n. 2, mar. 2017. ISSN 2177-4005. Disponível em: <https://online.unisc.br/seer/index.php/cinergis/ article/view/8286>. Acesso em: 29 mar. 2017. doi:http://dx.doi.org/10.17058/cinergis.v18i2.8286. 